\title{
Clinical value of interferon- $\gamma$ release assay in the diagnosis of active tuberculosis
}

\author{
LU AI ${ }^{*}$, PINNING FENG ${ }^{*}$, DUBO CHEN, SHAOQIAN CHEN and HONGXU XU \\ Department of Clinical Laboratory, The First Affiliated Hospital, \\ Sun Yat-Sen University, Guangzhou, Guangdong 510080, P.R. China
}

Received September 10, 2018; Accepted May 14, 2019

DOI: $10.3892 /$ etm.2019.7696

\begin{abstract}
Sensitivity and specificity of the interferon- $\gamma$ release test for active tuberculosis screening were evaluated. Due to the high-test cost of imported IGRAs, QFT-GIT and T-SPOT.TB, we applied a cheaper domestic TB-IGRA which was approved in China recently. We recruited 740 patients and performed tuberculosis interferon release test (IGRAs), detection of Mycobacterium tuberculosis $\mathrm{IgG}$ antibody (TB-IgG) and tuberculin skin test (TST). The sensitivity of the three methods are $90.8,40.0$ and $75.45 \%$, with specificity of 76.62, 74.47 and $72.27 \%$. The area under the ROC curve according to the value of T-N detected by IGRAs was 0.878 (95\% CI, 0.839-0.917), with the area under the curve for the diagnosis of active pulmonary tuberculosis and extrapulmonary tuberculosis being 0.839 and 0.841 respectively. The interferon- $\gamma$ release test seems to be superior to TST and TB-IgG as a screening tool for the detection of active tuberculosis in China.
\end{abstract}

\section{Introduction}

Tuberculosis is an infectious disease caused by Mycobacterium tuberculosis (MTB), which is a serious threat to people's health all over the world. According to the WTO's report, 1/3 of people infected with MTB, and about 1.7 million people die each year from tuberculosis and related diseases (1). Therefore, the rapid and accurate diagnosis of tuberculosis is critical for the treatment of tuberculosis. The sensitivity and specificity of traditional methods of detection (anti-acid stains smear, MTB culture, tuberculin skin test, tuberculosis antibody detection, MTB DNA detection) in the diagnosis of tuberculosis are limited. MTB isolation and culture test is the gold standard

Correspondence to: Dr Hongxu Xu, Department of Clinical Laboratory, The First Affiliated Hospital, Sun Yat-Sen University, 52 Zhongshan second Road, Guangzhou, Guangdong 510080, P.R. China

E-mail: xuhongxu@mail.sysu.edu.cn

*Contributed equally

Key words: tuberculosis, early diagnosis for the diagnosis of tuberculosis, but it takes a long time with little significance for early diagnosis, and it is difficult in sampling extra-pulmonary tuberculosis. In recent years, tuberculosis-specific $\mathrm{T}$ cell immunity in vitro detection method $\gamma$-interferon in vitro release test interferon- $\gamma$ release assay (IGRA), is gradually promoted and applied to clinic (2-4). This method is not affected by Bacillus Calmette-Guérin (BCG) vaccination and most non-tuberculosis mycobacterial infections in China, where BCG vaccination has a very good application prospects (5). In the present study, a domestic TB IFN- $\gamma$ release assay (HGN, Wuhan, China), was used as it is much cheaper than the imported IGRAs, QFT-GIT and T-SPOT.TB. It is licensed by the China Food and Drug Administration (CFDA), and a cheap and high-quality assay that will be constructive to the clinical application of IGRA and accumulating more clinical data for indicating its clinical value in developing countries with high TB burden. This study reviewed the results of interferon- $\gamma$ release assays (IGRAs) in 737 suspected tuberculosis patients in the above hospital from January 2016 to April 2016, and explored its diagnostic value and significance for active tuberculosis.

\section{Patients and methods}

Participants and samples. In total 740 patients with tuberculosis interferon release test admitted to The First Affiliated Hospital of Sun Yat-sen University (Guangzhou, China) in the period of January-April 2016, were recruited into the study. The age distribution was 1-96 years, including 737 cases of qualified specimens, 431 cases of male patients, hemolytic specimens 0 cases, lipid turbidity specimens 3 cases (Table I).

This study was reviewed and approved by the Institutional Review Board of The First Affiliated Hospital of Sun Yat-sen University according to the guidelines of Helsinki Declaration. The study was approved by the Ethics Committee of The First Affiliated Hospital, Sun Yat-Sen University. Patients who participated in this research had complete clinical data. The signed informed consents were obtained from the patients or the guardians.

Diagnostic criteria. Combined with clinical manifestations, clinical imaging, etiology and pathology data, the patients were divided into three categories: i) Confirmed active tuberculosis group: Smear positive for acid-fast bacilli, or MTB 
Table I. Demographic and clinical characteristics of the 737 patients.

\begin{tabular}{llll}
\hline Characteristics & \multicolumn{1}{c}{ Active TB } & Non-active TB & P-value \\
\hline Case number & 87 & 650 & \\
Age, median (range), years & $46(32-62)$ & $38(45-67)$ & 0.001 \\
Sex (male/female) & $58 / 29$ & $18(2.8 \%)$ & 0.062 \\
Evidence of previous TB (\%) & $2(2.3 \%)$ & & 0.353 \\
Blood tests (median, IQR) & & $1.70(1.06-2.18)$ \\
Lymphocytes $\left(x 10^{9} / 1\right)$ & $2.02(0.78-1.68)$ & $30(15-49)$ & 0.68 \\
ESR (mm/h) & $42(9-66)$ & $12.00(4.00-40.00)$ & 0.003 \\
CRP $(\mathrm{mg} / \mathrm{l})$ & $19.00(1.84-62.00)$ & $0.34(0.05-0.27)$ & 0.032 \\
PCT $(\mathrm{ng} / \mathrm{ml})$ & $8.08(0.05-74.25)$ & 0.009 \\
\hline
\end{tabular}

CRP, human C-reactive protein; PCT, procalcitonin; TB, tuberculosis

culture positive, pathological results suggesting granulomatous inflammation with coagulation necrosis; ii) Clinically diagnosed active tuberculosis group: The patients with suspected tuberculosis were treated with anti-tuberculosis therapy or follow-up observation, their features consistent of clinical and imaging findings of typical tuberculosis, but not confirmed by etiology and pathology, and clinicians excluded other non-tuberculous diseases; iii) Non-active tuberculosis group: pathological findings were clear for tumors, inflammation or other diseases, and excluded MTB infection, or clinical diagnosis for other diseases, by the corresponding treatment or follow-up observation (6). Clinicians excluded MTB infection through past history, clinical manifestations and laboratory examinations of the patients.

\section{Detection method}

IGRAs. For IGRAs, whole blood was collected from each patient and inoculated in three heparinized tubes of $1 \mathrm{ml}$ : each one containing TB antigens (ESAT-6 and CFP-10), a positive control tube containing phytohemagglutinin, and a null control. Blood samples were incubated for $20-24 \mathrm{~h}$ at $37^{\circ} \mathrm{C}$. Plasma samples were then harvested for IFN- $\gamma$ quantification by a single-step sandwich-type ELISA. The test was performed according to manufacturer's instructions (HyGeianey). Optical densities were interpreted by using specific software provided by the manufacturer. The result was considered to be positive if the IFN- $\gamma$ level after stimulation with TB antigens minus negative control was $\geq 0.35 \mathrm{IU} / \mathrm{ml}$ and $\geq 25 \%$ of the negative control. The test was considered negative if the IFN- $\gamma$ level was $<0.35 \mathrm{IU} / \mathrm{ml}$ (after subtraction of the negative control). The test result was considered to be indeterminate if the negative control was $\geq 8.0 \mathrm{IU} / \mathrm{ml}$ or the positive control minus negative control was $<0.5 \mathrm{IU} / \mathrm{ml}$ (Table II).

Detection of MTB $\operatorname{Ig} G$ antibody. Test operation and interpretation of the results were in strict accordance with the instructions of MTB IgG antibody detection kit (MP Biomedical Asia Pacific Limited).

TST. Patients were injected with $0.1 \mathrm{ml}$ of tuberculin [2 tuberculin units of pure protein derivatives (PPD)] in accordance with the American Thoracic Society guidelines. The skin induration was measured at 48-72 $\mathrm{h}$ after the
Table II. The result judgement of IGRAs.

\begin{tabular}{llcl}
\hline $\mathrm{N}(\mathrm{IU} / \mathrm{ml})$ & $\mathrm{P}-\mathrm{N}(\mathrm{IU} / \mathrm{ml})$ & \multicolumn{1}{c}{$\mathrm{T}-\mathrm{N}(\mathrm{IU} / \mathrm{ml})$} & $\begin{array}{c}\text { Result } \\
\text { judgement }\end{array}$ \\
\hline$\leq 8$ & Any value & $\geq 0.35$ and $\geq \mathrm{N} / 4$ & Positive \\
& $\geq 0.5$ & $<0.35$ & Negative \\
& $\geq 0.5$ & $\geq 0.35$ but $<\mathrm{N} / 4$ & \\
& $<0.5$ & $<0.35$ & Indeterminate \\
& $<0.5$ & $\geq 0.35$ but $<\mathrm{N} / 4$ & \\
& Any value & Any value & \\
\hline
\end{tabular}

IGRAs, interferon- $\gamma$ release assays

inoculation. The size of the induration equal or higher than $5 \mathrm{~mm}$ was considered positive. A previous BCG vaccination did not change the size limits for the tuberculin reaction. The result was considered to be invalid or not read, if the patient did not come to be measured or could not be contacted. All patients with a positive tuberculin test who had not been previously treated, received chemoprophylaxis with isoniazid for 6-9 months.

Statistical analysis. The statistical software package SPSS 22.0 (IBM Corp., Armonk, NY, USA) was used for data analysis to draw the ROC curve for the diagnosis value of IGRAs. Statistical comparisons were made by using the t-test to assess the difference between two proportions. Tests of statistical significance included the $95 \%$ confidence intervals of unadjusted relative risks. Values $<0.05$ were considered statistically significant. Sensitivity, specificity, positive predictive value, negative predictive value, positive likelihood ratio and negative likelihood ratio, and the $95 \%$ confidence interval of the corresponding indicators, were calculated to evaluate diagnostic performance of IGRAs by Medical software. The data were reviewed by laboratory quality-control staff, and data entry was performed by two independent persons. Most important, confirmed indeterminate samples were not enrolled into calculation in any equation. 
Table III. Diagnostic efficacy of results of IGRAs, TST and TB-IgG.

\begin{tabular}{lcccc}
\hline Test & $\begin{array}{c}\text { Sensitivity, } \% \\
(95 \% \mathrm{CI})\end{array}$ & $\begin{array}{c}\text { Specificity, } \% \\
(95 \% \mathrm{CI})\end{array}$ & $\begin{array}{c}\text { PPV, \% } \\
(95 \% \mathrm{CI})\end{array}$ & $\begin{array}{c}\text { NPV, } \% \\
(95 \% \mathrm{CI})\end{array}$ \\
\hline IGRAs & $90.80(82.68-95.95)$ & $76.62(73.17-79.82)$ & $34.18(33.33-34.53)$ & $98.66(97.44-99.40)$ \\
TST & $75.45(68.38-88.02)$ & $72.27(57.11-85.53)$ & $50.95(44.48-66.54)$ & $88.52(84.48-89.84)$ \\
TB-IgG & $40.00(26.41-54.2)$ & $74.47(59.65-86.06)$ & $11.16(6.25-19.85)$ & $96.75(85.25-99.85)$ \\
TST combined TB-IgG & $85.27(74.61-92.72)$ & $53.81(43.77-63.84)$ & $16.06(13.01-19.21)$ & $97.24(93.01-99.26)$ \\
\hline
\end{tabular}

IGRAs, interferon-gamma release assays; TST, tuberculin skin test, TB-IgG, Mycobacterium tuberculosis IgG antibody

Table IV. Comparison of sensitivity of IGRA test to different tuberculosis.

\begin{tabular}{lcc}
\hline Groups & $\begin{array}{c}\text { IGRAs } \\
\text { positive }\end{array}$ & $\begin{array}{c}\text { IGRAs } \\
\text { negative }\end{array}$ \\
\hline Pulmonary tuberculosis & 36 & 6 \\
Extra pulmonary tuberculosis & 34 & 2 \\
Tuberculous pleurisy & 14 & 1 \\
Lumbar tuberculosis & 4 & 1 \\
Intestinal tuberculosis & 8 & 0 \\
Tuberculosis of bone and joint & 2 & 0 \\
Other extra pulmonary tuberculosis & 6 & 0 \\
Pulmonary tuberculosis with & 9 & 0 \\
extrapulmonary tuberculosis & & \\
\hline
\end{tabular}

\section{Results}

Of the 737 patients who did receive IGRA, 87 cases were diagnosed with active tuberculosis (30 cases with confirmed active tuberculosis, 58 cases with clinically diagnosed active tuberculosis) and 650 cases non-active tuberculosis. The total sensitivity of IGRAs of active tuberculosis was $90.80 \%(79 / 87$, 95\% CI, 82.68-95.95), with specificity of 76.62\% (498/650, 95\% CI, 73.17-79.82), positive predictive value of $34.18 \%$ (95\% CI3.33-4.53) and the negative predictive value was $98.7 \%$ (498/506, 95\% CI, 97.44-99.40), the positive likelihood ratio was 3.88 , the negative likelihood compared to 0.12 . Table III shows the results of IGRAS, TST, TB-IgG test results, the sensitivity and specificity of the differences were statistically significant $(\mathrm{P}<0.05)$.

Among the 79 cases of active tuberculosis (including confirmed active tuberculosis and clinical diagnosed active tuberculosis), 8 were confirmed by pathogenicity as tuberculosis and the remaining 71 were smear-negative tuberculosis. The sensitivity of IGRAs to detect smear positive tuberculosis was $100 \%(8 / 8)$, and $88.73 \%(63 / 71)$ in the detection of smear-negative tuberculosis, and there was no significant difference $(\mathrm{P}>0.05)$.

The sensitivity of IGRAs to detect active pulmonary tuberculosis was $85.7 \%(36 / 42)$, and the sensitivity of extra-pulmonary tuberculosis (including tuberculous pleurisy, intestinal tuberculosis, lumbar tuberculosis, bone and joint tuberculosis and tuberculous meningitis) was $94.4 \%(34 / 36)$,

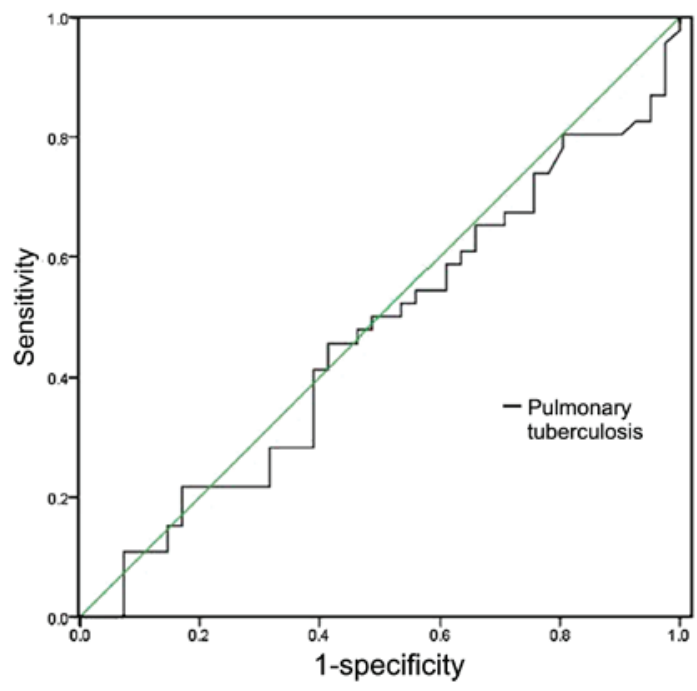

Figure 1. ROC curve of active tuberculosis.

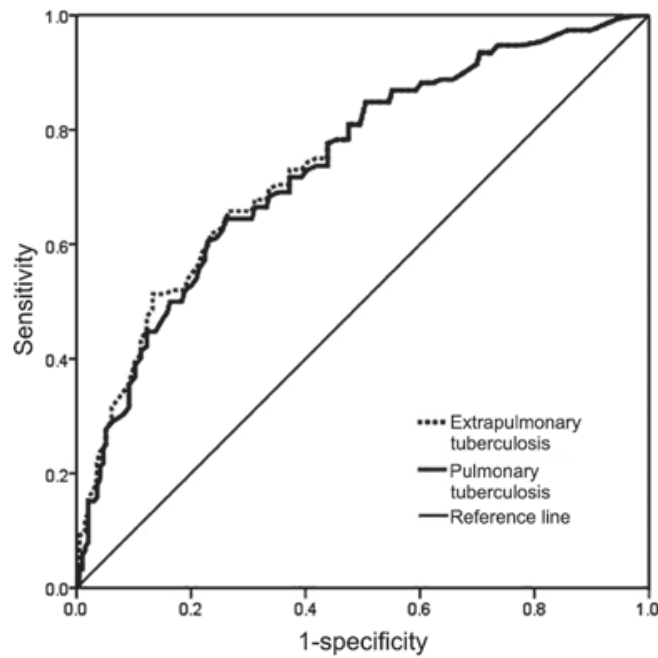

Figure 2. ROC curve of pulmonary tuberculosis and extrapulmonary tuberculosis.

the sensitivity of pulmonary tuberculosis with extrapulmonary tuberculosis was $100 \%$ (9/9). It was not statistically significant ( $\mathrm{P}>0.05)$ (Table IV).

According to the value of T-N detected by IGRAs, we performed ROC curve analysis (Fig. 1), and the area under the curve was 0.878 (95\% CI, 0.839-0.917), suggesting that 
IGRAs has a good application value in the diagnosis of active tuberculosis infection. $\mathrm{N}$ refers to a null control of interferon concentration in each patient, and $\mathrm{T}$ refers to the interferon concentration stimulated by tuberculosis antigen in the curve. Fig. 2 shows the ROC curve for the diagnosis of active pulmonary tuberculosis and extrapulmonary tuberculosis, with the area under the curve being 0.839 and 0.841 , respectively.

\section{Discussion}

Early diagnosis and treatment of tuberculosis has become a key factor in tuberculosis control (7-9). However, the positive rate of smear detection of acid-fast bacilli is very low, and the culture cycle of MTB is long, so they are not suitable for early diagnosis. The diagnostic value of microbiological methods is very limited. In this study, only 8 cases were smear positive for detection of acid-fast bacilli of 87 cases of active tuberculosis. TST can be used to diagnose tuberculosis quickly, but at the same time some antigenic components of the BCG pure protein derivative used in the TST are the same as those of BCG and most of the non-tuberculosis mycobacteria, and they are prone to cross-reactivity, making for high false-positive rate and low diagnostic specificity; while the response degree of the organism to PPD is affected by the state of the immune system, thus lacking sufficient sensitivity $(10,11)$. The detection of TB-IgG was influenced by the antigen processing, immunoassay method, and the immune response of the body, which have a great impact on the sensitivity and specificity of the test results (12).

IGRAs, developed in recent years based on the $\mathrm{T}$ cell immune response, have been shown to be more sensitive and specific in tuberculosis diagnosis. Meta-analysis of relevant literature and data from 1966 to 2006 by Menzies et al (13) showed that IGRA were more sensitive than TST in the diagnosis of tuberculosis. Domestic IGRAS research data show that the difference of the sensitivity and specificity is large, with the sensitivity of 53-98\% floating, specificity of $60-90 \%$ (or above), but most of the literature reported sensitivity and specificity greater than $70 \%$, showing that the specificity of IGRAs is better than PPD test (14). The results of this study show that the total sensitivity of IGRAs to detect active tuberculosis is similar to that reported in the literature, and the specificity is slightly lower.

At present, IGRA test is mainly used to assist diagnosis of MTB infection, and it cannot identify latent MTB infection and active tuberculosis. As the natural infection rate of tuberculosis is high in China, nearly $1 / 3$ of people have a history of MTB infection. In this study, non-active tuberculosis did not exclude latent MTB infection and old tuberculosis patients.

When the body is in a state of severe immunosuppression, such as immunodeficiency virus infection, decompensated liver cirrhosis, severe tuberculosis and non-fully developed childhood immune system whose $\mathrm{T}$ cell response is low, IGRAs may be false-negative, and the elderly, body mass index $<16.0 \mathrm{~kg} / \mathrm{m}^{2}$ may also result in false-negatives (15). The positive reaction of interferon $-\gamma$ release test can also be changed in anti-tuberculosis treatment. In this study, there were 8 cases of false-negative: 1 case was followed up for more than 2 years after renal transplantation, with long-term use of anti-rejection drugs; 1 case of systemic lupus erythematosus active patient, who accepted repeated hormone shock treatment; 1 case of lumbar spinal tuberculosis patient who was treated with anti-tuberculosis before IGRA test; but there are still five false-negative results which cannot be explained. In the literature, the IGRAs trial has a high negative predictive value, but negative results are not yet possible to exclude the possibility of active tuberculosis, especially in immunosuppressed population (16). Now researchers use pleural effusion, cerebrospinal fluid as IGRA test specimens, but whether the negative results are more valuable, is worth exploring.

In summary, IGRA have high sensitivity and good specificity in the diagnosis of active tuberculosis, but cannot identify latent MTB infection and old tuberculosis, which is approved by the US Food and Drug Administration for the diagnosis of MTB infection rather than active tuberculosis. The results of this study confirm that IGRAs can be widely used in the diagnosis of MTB infection in China which has a high natural infection rate of MTB, and its higher negative predictive value can provide an important reference for the diagnosis and treatment of clinical tuberculosis. Moreover, due to the low cost and high quality of the domestic TB-IGRA, it can be a useful tool in MTB infection detection in low-income countries with TB endemics.

\section{Acknowledgements}

Not applicable.

\section{Funding}

No funding was received.

\section{Availability of data and materials}

The datasets used and/or analyzed during the present study are available from the corresponding author on reasonable request.

\section{Authors' contributions}

LA and PF drafted the manuscript. LA, PF and DC were mainly devoted to collecting and interpreting the data. LA, SC and HX revised it critically for important intellectual content. PF, DC and HX were responsible for the conception and design of the study. All the authors read and approved the final manuscript.

\section{Ethics approval and consent to participate}

The study was approved by the Ethics Committee of The First Affiliated Hospital, Sun Yat-Sen University (Guangzhou, China). Patients who participated in this research had complete clinical data. The signed informed consents were obtained from the patients or the guardians.

\section{Patient consent for publication}

Not applicable.

\section{Competing interests}

The authors declare that they have no competing interests. 


\section{References}

1. World Health Organization (WHO): Global tuberculosis control: Surveillance, financing WHO report 2005. World Health Organization (WHO/HTM/TB/2005.349) Geneva. https://apps who.int/iris/bitstream/handle/10665/144569/9241562919_eng.pdf? sequence $=1$.

2. Zhang X, Sun Y, He C, Qiu X, Zhou D, Ye Z, Long Y, Tang T, $\mathrm{Su} X$ and $\mathrm{Ma} \mathrm{J}$ : The immune characterization of interferon- $\beta$ responses in tuberculosis patients. Microbiol Immunol 62 : 281-290, 2018

3. Andersen P, Munk ME, Pollock JM and Doherty TM: Specific immune-based diagnosis of tuberculosis. Lancet 356: 1099-1104, 2000.

4. Zhang S, Shao L, Mo L, Chen J, Wang F, Meng C, Zhong M, Qiu L, $\mathrm{Wu}$ M, Weng X, et al: Evaluation of gamma interferon release assays using Myeobacterium tuberculosis antigens for diagnosis of latent and active tuberculosis in Mycobacterium bovis BCG-vaccinated populations. Clin Vaccine lmmunol 17: 1985-1990, 2010.

5. Sayyahfar S, Davoodzadeh F, Hoseini R, Rahimzadeh N and Otukesh H: Comparison of tuberculin skin test and interferon gamma release assay for diagnosis of latent tuberculosis infection in pediatric candidates of renal transplantation. Pediatr Transplant 22: e13148, 2018.

6. Ministry of Health of the People's Republic of China: Diagnostic criteria for pulmonary tuberculosis. People's Health Publishing House, Beijing, pp1-16, 2008 (In Chinese).

7. Diel R, Loddenkemper R and Nienhaus A: Evidence-based comparison of commercial interferon-gamma release assays for detecting active TB: A metaanalysis. Chest 137: 952-968, 2010.

8. Laurenti P, Raponi M, de Waure C, Marino M, Ricciardi W and Damiani G: Performance of interferon- $\gamma$ release assays in the diagnosis of confirmed active tuberculosis in immunocompetent children: A new systematic review and meta-analysis. BMC Infect Dis 16:131, 2016.
9. Galloway KM and Parker R: Could an increase in vigilance for spinal tuberculosis at primary health care level, enable earlier diagnosis at district level in a tuberculosis endemic country? Afr J Prim Health Care Fam Med 10: e1-e9, 2018.

10. Thwaites GE: Advances in the diagnosis and treatment of tuberculous meningitis. Curr Opin Neurol 26: 295-300, 2013

11. Kusum S, Aman S, Pallab R, Kumar SS, Manish M, Sudesh P, Subhash V and Meera S: Multiplex PCR for rapid diagnosis of tuberculous meningitis. J Neurol 258: 1781-1787, 2011.

12. Qin L, Zhang L, Zhang Y, Shi X, Zhang Y and Liu X: Diagnostic value of T-Cell interferon- $\gamma$ release assays on cerebrospinal fluid for tuberculous meningitis. PLoS One 10: 0141814, 2015.

13. Menzies D, Pai M and Comstock G: Meta-analysis: New tests for the diagnosis of latent tuberculosis infection: Areas of uncertainty and recommendations for research. Ann Inter Med 146: 340-354, 2007.

14. Editorial Board of Chinese Journal of Tuberculosis and Respiratory Medicine, Chinese Medical Association: The application of interferon gamma release test in China. Chin J Tuberculosis Respir Dis 10: 37, 2014 (In Chinese).

15. Hang NT, Lien LT, Kobayashi N, Shimbo T, Sakurada S, Thuong PH, Hong LT, Tam DB, Hijikata M, Matsushita I, et al: Analysis of factors lowering sensitivity of interferon- $\gamma$ release assay for tuberculosis. PLoS One 6: e23806, 2011.

16. Maze M and Beckert L: Active tuberculosis (TB) with a negative interferon gamma release assay: Failure of this test to rule out TB. N Z Med J 126: 85-87, 2013

(c) (i) () This work is licensed under a Creative Commons

cc) $\mathrm{EY}$ NO $\mathrm{ND}$ Attribution-NonCommercial-NoDerivatives 4.0 International (CC BY-NC-ND 4.0) License. 